\title{
Optimization of Percutaneous Biopsy for Diagnosis and Pretreatment Risk
}

\section{Assessment of Neuroblastoma}

Short Running Title: Percutaneous Biopsy for Neuroblastoma

Richard E. Overman, MD ${ }^{1}$; Tanvi T Kartal, BS ${ }^{1}$; Aaron J Cunningham, MD ${ }^{2}$; Elizabeth A Fialkowski, $\mathrm{MD}^{2}$; Bindi J Naik-Mathuria, MD, $\mathrm{MPH}^{3}$; Sanjeev A Vasudevan, $\mathrm{MD}^{3}$; Marcus M Malek, MD ${ }^{4}$; Ranjeet Kalsi, DO ${ }^{4}$; Hau D. Le, MD ${ }^{5}$; Linda Cherney Stafford, MPH Timothy B Lautz, MD ${ }^{6}$; Benjamin T Many, $\mathrm{MD}^{6}$; Rachel E Jones ${ }^{6}$; Andreana Bütter, $\mathrm{MD}^{7}$; Jacob Davidson ${ }^{7}$; Andrew Williams ${ }^{7}$; Roshni Dasgupta, MD, $\mathrm{MPH}^{8}$; Jana Lewis ${ }^{8}$; Misty Troutt $^{8}$; Jennifer H Aldrink, MD ${ }^{9}$; Sara A Mansfield, MD, MS ${ }^{9}$; Dave R Lal, MD, MPH ${ }^{10}$; Jerry Xiao ${ }^{10}$; Rebecka L. Meyers, MD ${ }^{11}$; Scott S Short, $\mathrm{MD}^{11}$; Erika A. Newman, MD ${ }^{1}$

${ }^{1}$ Department of Surgery, Division of Pediatric Surgery, C. S. Mott Children's Hospital, The University of Michigan, Ann Arbor, MI

${ }^{2}$ Department of Surgery, Division of Pediatric Surgery, Oregon Health and Science University

${ }^{3}$ Department of Surgery, Texas Children's Hospital, Baylor College of Medicine

${ }^{4}$ Division of Pediatric General and Thoracic Surgery, UPMC Children's Hospital of Pittsburgh, University of Pittsburgh School of Medicine, Pittsburgh, PA

${ }^{5}$ Division of Pediatric Surgery, American Family Children's Hospital, University of Wisconsin School of Medicine and Public Health

${ }^{6}$ Division of Pediatric Surgery, Ann \& Robert H Lurie Children's Hospital of Chicago, Northwestern University

${ }^{7}$ Division of Pediatric Surgery, Children's Hospital, London Health Sciences Centre, Western University

${ }^{8}$ Division of Pediatric General and Thoracic Surgery, Cincinnati Children's Hospital Medical Center, Cincinnati, $\mathrm{OH}$

${ }^{9}$ Division of Pediatric Surgery, Department of Surgery, Nationwide Children's Hospital, The Ohio State University College of Medicine

${ }^{10}$ Division of Pediatric Surgery, Medical College of Wisconsin, Children's Hospital of Wisconsin

${ }^{11}$ Division of Pediatric Surgery, Primary Children's Hospital, University of Utah, Salt Lake City, UT

This is the author manuscript accepted for publication and has undergone full peer review but has not been through the copyediting, typesetting, pagination and proofreading process, which may lead to differences between this version and the Version of Record. Please cite this article as doi: $10.1002 / \mathrm{pbc} .28153$.

This article is protected by copyright. All rights reserved. 


\section{Corresponding Author:}

Erika A. Newman, MD

Associate Professor of Surgery

C.S. Mott Children's Hospital

The University of Michigan

Ann Arbor, MI 48109

phone: 734-764-6482

fax: 734-232-8667

eanewman@med.umich.edu

\section{Word Counts:}

Abstract: 248

Main Text: 2,309

\section{Tables: 4}

Figures: 1

Supporting Information: 0 
Abbreviations Table:

\begin{tabular}{|c|c|}
\hline IB & Incisional Biopsy \\
\hline PCNB & Percutaneous Core Needle Biopsy \\
\hline INRG & International Neuroblastoma Risk Group \\
\hline PSORC & Pediatric Surgical Oncology Research Collaborative \\
\hline COG & Children's Oncology Group \\
\hline $\mathrm{LOH}$ & Loss of Heterozygosity \\
\hline NPO & Nil per Os \\
\hline PCA & Patient Controlled Analgesia \\
\hline
\end{tabular}

Key Words: Neuroblastoma; neuroblastoma Biology; Tumor biology; Surgery; Solid Tumors; Percutaneous biopsy

Data Availability Statement: Author elects not to share data

This work was presented as an abstract at the American Pediatric Surgery Association meeting on May 21, 2019, under the name Percutaneous Core Needle Biopsy for Initial Diagnostic Management of Neuroblastoma
Abstract
Background

Image-guided percutaneous core needle biopsy (PCNB) is increasingly utilized to diagnose solid tumors. The objective of this study is to determine whether PCNB is adequate for modern biologic characterization of neuroblastoma.

Procedure

A multi-institutional retrospective study was performed by the Pediatric Surgical Oncology Research Collaborative on children with neuroblastoma at 12 institutions over a 3-year period. Data collected included demographics, clinical details, biopsy technique, 
complications, and adequacy of biopsies for cytogenetic markers utilized by the Children's Oncology Group for risk stratification.

Results

243 children were identified with a diagnosis of neuroblastoma: $79(32.5 \%)$ tumor excision at diagnosis, 94 (38.7\%) open incisional biopsy (IB), and 70 (28.8\%) PCNB. Compared to IB, there was no significant difference in ability to accurately obtain a primary diagnosis by PCNB $(95.7 \%$ vs $98.9 \%, p=0.314)$ or determine MYCN copy number $(92.4 \%$ vs $97.8 \%, p=$ 0.111). The yield for loss of heterozygosity and tumor ploidy was lower with PCNB versus IB (56.1\% vs $90.9 \%, \mathrm{p}<0.05$; and $58.0 \%$ vs. $88.5 \%$, p $<0.05)$. Complications did not differ between groups $(2.9 \%$ vs $3.3 \%, p=1.000)$, though the PCNB group had fewer blood transfusions and lower opioid usage. Efficacy of PCNB was improved for loss of heterozygosity when a pediatric pathologist evaluated the fresh specimen for adequacy.

Conclusions

PCNB is a less invasive alternative to open biopsy for primary diagnosis and MYCN oncogene status in patients with neuroblastoma. Our data suggests that PCNB could be optimized for complete genetic analysis by standardized protocols and real-time pathology assessment of specimen quality. 
Introduction

Neuroblastoma is the most common extracranial solid tumor in children, with approximately 700 new cases diagnosed each year in the United States. ${ }^{1}$ Tumor biologic and genetic characteristics are heterogeneous, and therapeutic decisions depend on appropriate tissue sampling and risk classification. ${ }^{2} \mathrm{MYCN}$ amplification and segmental chromosomal alterations are important indicators that drive treatment decisions and determine overall disease risk. High-risk patients receive intense cytotoxic chemotherapy, while some patients with low-risk disease can be managed with observation alone. The optimal technique for tissue acquisition minimizes patient morbidity and obtains adequate tissue for complete biologic analysis and pretreatment assessment. Tissue biopsy has traditionally been achieved with open incisional biopsy (IB), however the use of image-guided percutaneous core needle biopsy (PCNB) is increasingly utilized for pediatric surgical patients. PCNB offers substantial advantages including lower procedural costs, fewer complications, and avoids the risks of open surgery. ${ }^{3-5}$

The International Neuroblastoma Risk Group (INRG) Task Force developed guidelines for neuroblastoma risk stratification which include age, radiographic imaging factors, tumor stage, and tumor genetic profile. ${ }^{2,6}$ There is conflicting data regarding the efficacy of PCNB for cytogenetic analysis, relying primarily on single-institutional data. Several reports have demonstrated comparable accuracy between PCNB and IB, while others challenge the full diagnostic yield of PCNB. ${ }^{7--13}$ Significant variability exists regarding which patients may benefit from PCNB, and standard surgical techniques have not been defined for PCNB. Several small studies have demonstrated lower or similar complication rates, but detailed procedural and post-operative outcomes are lacking. ${ }^{5,7,8}$ What remains unknown are the precise technical practices that may optimize the efficacy of PCNB required for current treatment protocols and for clinical trial enrollment. 
To improve efficacy of PCNB, the Pediatric Surgical Oncology Research

Collaborative (PSORC) conducted a multi-institutional, retrospective study to evaluate procedural details and outcomes of PCNB for neuroblastoma. Differences in technical practices and peri-operative outcomes were examined. These results set precedence for a collaborative multidisciplinary approach to establish technical standards for PCNB for pediatric solid tumors.

Methods

Twelve surgical centers in North America participated and contributed data to this study. All patients under the age of 18 with a new diagnosis of neuroblastoma between June 1, 2014 and November 1, 2017 were included. Data extracted from chart review included demographic information, initial imaging modalities, characteristics of the mass (including size, position relative to critical anatomic structures, and evidence of necrosis on initial imaging), biopsy procedure type, technical details regarding the biopsy, complications from the biopsy procedure, diagnostic yield (including cytogenetic markers recommended by the Children's Oncology Group [COG] protocols for pre-treatment risk stratification and clinical trial enrollment), clinical information detailing post-biopsy admission, and pain medication requirements. Data was de-identified and entered into a central secure database.

Data was stratified according to type of initial surgical intervention: tumor excision, IB or PCNB. Patients who had tumor excision at diagnosis were not included in the comparative analysis. The primary outcome was success in establishing the diagnosis of neuroblastoma at the initial biopsy. Secondary outcomes included success in obtaining the critical cytogenetic markers (MYCN copy number, loss of heterozygosity (LOH), and tumor ploidy) needed for risk stratification; length of stay and NPO after biopsy; pain management profile (analgesics, opioid use, requirement of patient controlled analgesia [PCA]); transfusion requirements; need for repeat biopsy; and complications. Data was collected on 
institutional site, surgical technique (number of core needle samples obtained, size/needle gauge of core needle biopsy, surgeon vs radiologist performing biopsy) and on pathologic characteristics (pathologist real-time tissue analysis, tumor size, and tumor necrosis).

Study data was collected using the REDCap electronic data access tools, hosted at Cincinnati Children's Hospital Medical Center, and converted to STATA for analysis. ${ }^{14}$ Sample characteristics are reported as number of observations and percentages for categorical variables, and median and range for continuous variables. Comparisons between age, gender, adequacy of biopsy for diagnosis and cytogenetic analysis, complication rates, and need for post-operative analgesia were assessed by Chi-squared or Fisher's Exact tests as appropriate. To account for the multi-center nature of the study design, mixed effect logistic regression analysis was used to assess the effects of tumor size, biopsy needle size, number of biopsies taken, presence of real-time pathologic review during the procedure, and necrosis on imaging on successful diagnosis and tumor characterization. All analyses were conducted in STATA v15.1, STATACorp, College Station, TX, ( $\mathrm{p}$ value less than 0.05 considered statistically significant).

Results

A total of 243 children with newly diagnosed neuroblastoma were identified from 12 institutions. Seventy-nine patients had tumor excision as the primary procedure and were therefore excluded from the comparative analysis. The remaining 164 were divided into two groups by initial biopsy type: 70 PCNB and 94 IB. (Figure 1) The only significant baseline demographic differences between the IB and PCNB groups was a higher proportion of male patients with IB. (Table 1)

There was no difference between PCNB and IB in ability to determine the primary diagnosis of neuroblastoma $(95.7 \%$ vs $98.9 \%, \mathrm{p}=0.314)$ or $\mathrm{MYCN}$ copy number $(92.4 \%$ vs $97.8 \%, \mathrm{p}=$ 
0.111). IB was more successful at obtaining adequate samples for $\mathrm{LOH}$ (IB $91.8 \%$ vs PCNB $60.8 \%, \mathrm{p}<0.05$ ) and tumor ploidy analysis (IB $89.1 \%$ vs PCNB $57.8 \%, \mathrm{p}<0.05$ ). (Table 2)

Overall complication rate was low in both groups (PCNB 2.9\% vs IB $3.33 \%, \mathrm{p}=$ 0.624). Patients undergoing IB were more likely to require at least one blood transfusion during their admission $(40.4 \%$ vs $15.7 \%, \mathrm{p}=0.001)$. Analgesia requirement, including opioid administration and the need for patient controlled analgesia were all lower in the PCNB group than the IB group. Patients undergoing PCNB were less likely to require hospital admission post-operatively than patients undergoing IB $(80.0 \%$ vs $96.8 \%, p=0.001)$. Length of stay and total days kept NPO did not differ between the two groups. (Table 3)

Mixed effect logistic regression modeling was employed to evaluate for factors that contributed to higher diagnostic yield of the primary outcomes (primary diagnosis, MYCN copy number, $\mathrm{LOH}$ and tumor ploidy), while controlling for inter-institutional variability. There were no statistically significant relationships observed between core needle sizes, the numbers of core samples obtained, or usage of ultrasound guidance for biopsy and diagnostic yield. The impact of tumor size and tumor necrosis on imaging were also analyzed, and had no impact on diagnostic yield.

Finally, we analyzed the effect that different personnel had on the diagnostic yield for patients undergoing PCNB. Of the seventy PCNB reviewed, 14 (20\%) were performed by surgeons, while $56(80 \%)$ were performed by interventional radiologists. There were no differences in diagnostic yield of any of the primary outcomes between surgeons and interventional radiologists. Surgeons were more likely than radiologists to use ultrasound guidance during percutaneous biopsy $(100 \%$ vs $71.1 \%, \mathrm{p}=0.020)$. Masses biopsied by surgeons were larger than those biopsied by radiologists (median largest mass size $7.4 \mathrm{~cm}$ vs $3.4 \mathrm{~cm}, \mathrm{p}=0.002)$, but did not have significantly more necrosis present on initial imaging 
$(28.6 \%$ biopsied by surgeons with necrosis, vs $10.5 \%$ by pathologists, $p=0.189)$. Six of the twelve centers had a pediatric pathologist present during PCNB to aid in ensuring adequacy of tissue specimen. Since diagnostic yield and MYCN copy number were already adequate, the presence of a pathologist did not alter these categories. While there were not effects on ability to obtain tumor ploidy $(60.0 \%$ vs $55.8 \%, \mathrm{p}=0.791)$, the rate of adequacy for $\mathrm{LOH}$ was improved with a pathologist present to evaluate the gross specimen $(92.8 \%$ vs $48.6 \%, p=$ 0.008). (Table 4)

\section{Discussion}

The utilization of PCNB for diagnosis and pathologic characterization of neuroblastoma and other pediatric solid tumors has increased in recent years. ${ }^{5,15}$ Several reports have found similar efficacy in diagnosis rate between percutaneous and open biopsy methods, while others have reported decreased diagnostic yield with PCNB. ${ }^{7,8,10,11}$ The INRG classification system outlines pre-treatment risk groups to stratify patients based on probability of disease progression and recurrence. This classification system requires age, histologic factors, biologic and genetic characterization (MYCN amplification, DNA ploidy, and loss of heterozygosity at 11q). ${ }^{1,16}$ Small pilot studies have confirmed the ability of PCNB to obtain a diagnosis in neuroblastoma, but these have not uniformly included a robust evaluation of ability to obtain adequate tissue for complete genomic assessment.

Our study compares the efficacy of PCNB and IB in both diagnosing neuroblastoma and obtaining complete genomic assessment of MYCN, LOH, and tumor ploidy. The ability of PCNB to determine the primary diagnosis of neuroblastoma and determine MYCN copy number was equivalent to IB. However, IB was superior to PCNB in determination of tumor ploidy status and $\mathrm{LOH}$ at 11q. This is an important issue to address as $\mathrm{LOH}$ and ploidy determinations are essential in accurate risk classification. ${ }^{17}$ Analysis of the technical aspects of PCNB was undertaken in order to identify practices that optimize tissue samples for 
cytogenetic testing. Our results suggest that peri-procedural qualitative review of the gross specimen by a pediatric pathologist is associated with increased complete genetic assessment, including $\mathrm{LOH}$. This is likely due to the ability of the pathologist to determine which core specimens contained viable appearing tissue versus necrotic cores. The current logistic regression analysis did not demonstrate significant association between number of cores obtained, size of percutaneous needle utilized, or tumor-specific features on imaging that increase diagnostic yield, although literature for other pediatric solid tumors have recommended the range of $10--20$ cores be obtained at minimum. ${ }^{18,19}$ This is likely due to the relatively small sample sizes in subgroups within this analysis as a result of the many different technical steps, cores obtained, needle sizes, and equipment utilized both interinstitutional and within the collaborative multicenter group. These variable results and previous retrospective studies set premise for larger prospective studies to further optimize successful PCNB techniques. Despite the lower number of PCNB patients with successful LOH (60.8\%) and tumor ploidy assessment (57.8\%), there was not a statistically significant increase in the number of patients requiring repeat biopsies (PCNB 7.1\% vs IB 1.1\%, p= 0.085). (Table 3) This implies that the information received from PCNB is adequate for treatment decisions in select patients.

This study and others have shown that PCNB is associated with lower morbidity than open biopsy. While complications in both groups were low, there was significantly less analgesia use in patients undergoing PCNB, as well as a lower incidence of blood transfusions. A large majority of patients in both groups were admitted post-operatively, and length of stay was significantly longer than would be expected from biopsy procedures alone. This is likely due to the frequent practice of admission, radiologic imaging for staging, multidisciplinary consultation, and induction chemotherapy immediately following biopsy, and it is therefore difficult to draw definitive conclusions regarding length of stay data. The majority of PCNB 
were performed by interventional radiologists $(80 \%)$, though some were performed by surgeons (20\%). There were no significant differences in the diagnostic yield of biopsies obtained by either specialty in this study. Because both specialties perform PCNB depending on institution, a standardized technical approach could strengthen success of genetic analysis.

The logistic regression in our analysis was not able to demonstrate differences in diagnostic ability with respect to cores obtained and needle size, and subgroup analysis was hindered by significant missing data in both groups -- $24 \%$ missing in the interventional radiologist performed group, and $43 \%$ in the surgeon-performed group. As a result, we felt that reporting the characteristics of the successful percutaneous biopsies in our group might aid clinicians in their decision making. Of the 52 patients who underwent percutaneous biopsy, 21 cases $(40.3 \%)$ had tissue adequate for all primary diagnosis as well as genomic assessment of MYCN, LOH, and tumor ploidy. In this group, 38\% underwent biopsy with an 18 gauge need, $19 \%$ with a 16 gauge needle, and $14 \%$ with a 14 gauge needle or larger $(29 \%$ had no needle size recorded). The median number of cores obtained in this group was 9, with an interquartile range of 6--30.

This study has several limitations. Despite demographically similar groups, the retrospective nature of the study does not allow for randomized control groups and therefore we are unable to control for inherent bias. Additionally, data was not collected regarding rationale for the choice between percutaneous and open biopsy, and it may be that tumors which were smaller or more difficult to access percutaneously preferentially underwent open biopsy. Currently, there is no standard of care or protocol for PCNB technique, size of needle utilized, or number of cores obtained. There are also not current recommendations or requirements for pathologic review during the procedure for pretreatment risk stratification or clinical trial enrollment. While logistic regression attempted to evaluate trends in these areas, there was missing data regarding the sizes of needles $(28.9 \%$ missing $)$ and number of cores obtained 
( $9.6 \%$ missing), and this may confound the ability to detect a statistically significant

difference. These limitations point to the need for a controlled, prospective, study for quality improvement. Finally, and perhaps importantly, we are not able to comment on the relative success of IB and PCNB in securing tissue for banking and experimental biologic research.

Despite these limitations, this data demonstrates that there are significant benefits to PCNB in patients undergoing evaluation for neuroblastoma. PCNB was successful at obtaining histologic diagnosis and MYCN copy number. In select patients, this may be adequate to inform necessary pre-treatment decisions and spare patients the morbidity associated with open biopsy. PCNB was successful in obtaining the complete profile of genetic testing in about 60 percent and we hypothesize that this could be improved with vigilance, standardization of biopsy technique, and real-time pathology assessment of tissue.

\section{Conclusion}

PCNB for neuroblastoma is a feasible alternative to IB for select patients with neuroblastoma, but in current practice remains inadequate for obtaining important molecular data in a significant fraction of patients. Standardization of technical aspects that improve the yield of important cytogenetic information, including real time pathology review, represents an important opportunity to advance surgical care of children with neuroblastoma and other pediatric solid tumors.

Conflicts of Interest Statement:

The authors have no conflicts of interest relevant to this work.

Acknowledgements:

None 
References

1. Newman EA, Abdessalam S, Aldrink JH, et al. Update on neuroblastoma. J Pediatr Surg. 2019;54(3):383-389. doi:10.1016/j.jpedsurg.2018.09.004

2. Monclair T, Brodeur GM, Ambros PF, et al. The International Neuroblastoma Risk Group (INRG) staging system: An INRG Task Force report. J Clin Oncol. 2009;27(2):298-303. doi:10.1200/JCO.2008.16.6876

3. Kim J, Sun Z, Adam MA, et al. Predictors of nodal metastasis in pediatric differentiated thyroid cancer. J Pediatr Surg. 2017;52(1):120-123. doi:10.1016/J.JPEDSURG.2016.10.033

4. Pohlig F, Kirchhoff C, Lenze U, et al. Percutaneous core needle biopsy versus open biopsy in diagnostics of bone and soft tissue sarcoma: a retrospective study. Eur J Med Res. 2012;17(1):29. doi:10.1186/2047-783X-17-29

5. Weldon CB, Madenci AL, Tiao GM, et al. Evaluation of the diagnostic biopsy approach for children with hepatoblastoma: A report from the Children's Oncology Group AHEP 0731 Liver Tumor Committee. J Pediatr Surg. 2019;(xxxx). doi:10.1016/j.jpedsurg.2019.05.004

6. Simon T, Matthay KK, Castel V, et al. The International Neuroblastoma Risk Group (INRG) Classification System: An INRG Task Force Report. J Clin Oncol. 2008;27(2):289-297. doi:10.1200/jco.2008.16.6785

7. Mullassery D, Sharma V, Salim A, et al. Open versus needle biopsy in diagnosing neuroblastoma. J Pediatr Surg. 2014;49(10):1505-1507. doi:10.1016/j.jpedsurg.2014.05.015

8. Hassan SF, Mathur S, Magliaro TJ, et al. Needle core vs open biopsy for diagnosis of 
intermediate- and high-risk neuroblastoma in children. J Pediatr Surg. 2012;47(6):1261-1266. doi:10.1016/j.jpedsurg.2012.03.040

9. Campagna G, Rosenfeld E, Foster J, et al. Evolving biopsy techniques for the diagnosis of neuroblastoma in children. J Pediatr Surg. 2018;53(11):2235-2239. doi:10.1016/j.jpedsurg.2018.04.012

10. Gupta A, Kumar A, Walters S, Chait P, Irwin MS, Gerstle JT. Analysis of needle versus open biopsy for the diagnosis of advanced stage pediatric neuroblastoma. Pediatr Blood Cancer. 2006;47(7):875-879. doi:10.1002/pbc.20666

11. Hoffer A, Chung T, Diller L, Kozakewich H, Fletcher A, Shamberger C. Percutaneous biopsy for prognostic testing of neuroblastoma. Radiology. 1996;200:213-216. doi:10.1148/radiology.200.1.8657913

12. Metz T, Heider A, Vellody R, et al. Image-guided percutaneous core needle biopsy of soft-tissue masses in the pediatric population. Pediatr Radiol. 2016;46(8):1173-1178. doi:10.1007/s00247-016-3571-5

13. Sklair-Levy M, Lebensart PD, Applbaum YH, et al. Percutaneous image-guided needle biopsy in children - Summary of our experience with 57 children. Pediatr Radiol. 2001;31(10):732-736. doi:10.1007/s002470100533

14. Harris PA, Thielke R, Gonzalez N, Conde JG, Taylor R, Payne J. Research electronic data capture (REDCap)---A metadata-driven methodology and workflow process for providing translational research informatics support. J Biomed Inform. 2008;42(2):377-381. doi:10.1016/j.jbi.2008.08.010

15. Campagna G, Rosenfeld E, Foster J, et al. Evolving biopsy techniques for the diagnosis of neuroblastoma in children. J Pediatr Surg. 2018. 
doi:10.1016/j.jpedsurg.2018.04.012

16. Riley RD, Heney D, Jones DR, et al. A systematic review of molecular and biological tumor markers in neuroblastoma. Clin Cancer Res. 2004;10(1 Pt 1):4-12.

http://www.embase.com/search/results?subaction $=$ viewrecord\&from $=$ export\&id $=$ L36005421\%5Cnhttp://dx.doi.org/10.1016/S0959-8049(02)00500-

2\%5Cnhttp://elvis.ubvu.vu.nl:9003/vulink?sid = EMBASE\&issn = 09598049\&id $=$ doi:10.1016\%2FS0959-8049\%2802\%2900500-2\&atitle = A+syste.

17. Schleiermacher G, Janoueix-Lerosey I, Ribeiro A, et al. Accumulation of segmental alterations determines progression in neuroblastoma. J Clin Oncol. 2010;28(19):31223130. doi:10.1200/JCO.2009.26.7955

18. Lim IIP, Bondoc AJ, Geller JI, Tiao GM. Hepatoblastoma---The Evolution of Biology, Surgery, and Transplantation. Children. 2018;6(1):1. doi:10.3390/children6010001

19. López-Terrada D, Alaggio R, De Dávila MT, et al. Towards an international pediatric liver tumor consensus classification: Proceedings of the Los Angeles COG liver tumors symposium. Mod Pathol. 2014;27(3):472-491. doi:10.1038/modpathol.2013.80 
Figure Legend:

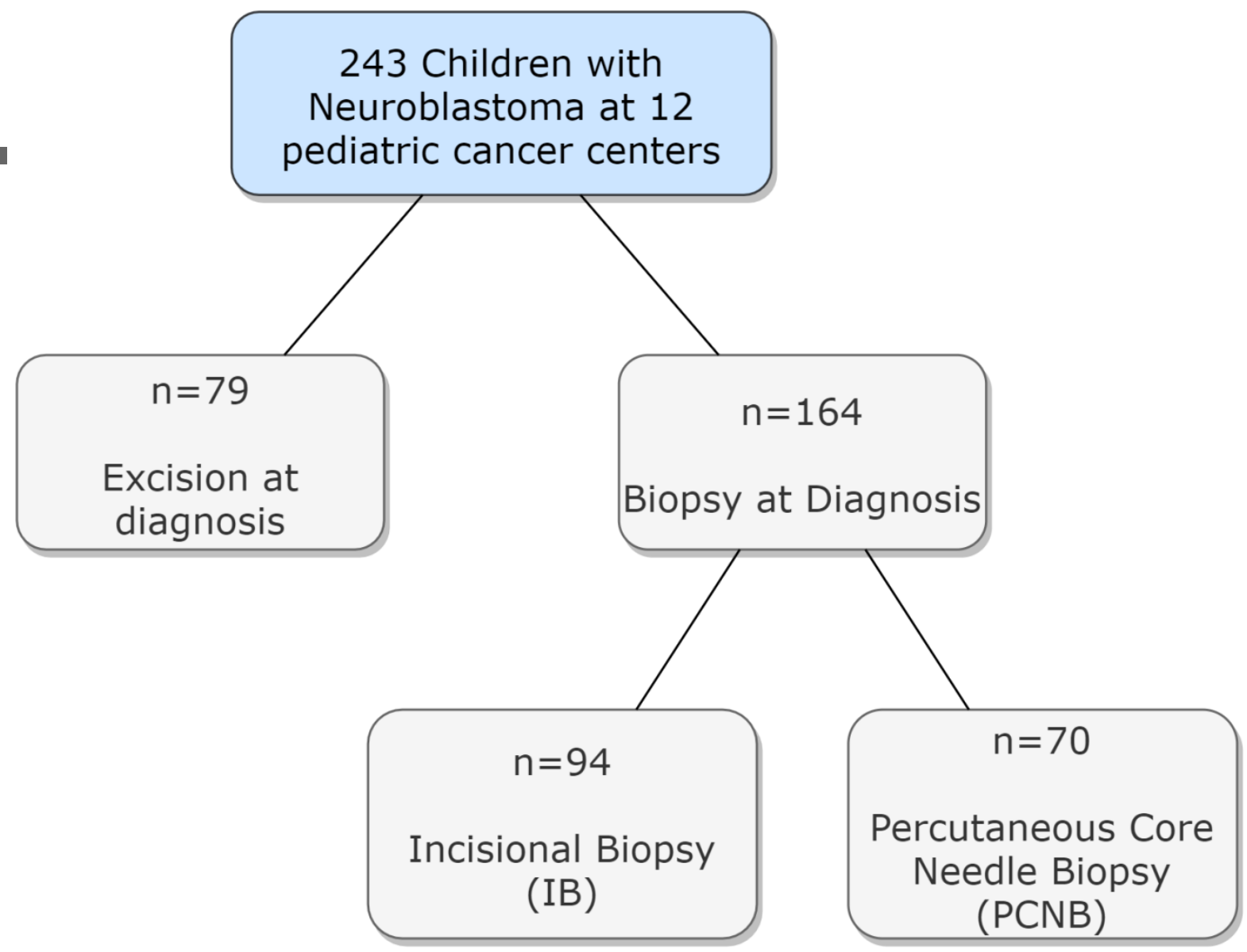

FIGURE 1: Details of patient selection and exclusion 
TABLE 2: Diagnostic yield of biopsies

Sample Adequate for: Percutaneous Core Needle Biopsy Incisional Biopsy
$(n, \%)$
$n=70$
$n=94$

p-value

\begin{tabular}{r|c|c|c}
\hline Primary Diagnosis & $67(95.7 \%)$ & $93(98.9 \%)$ & 0.314 \\
\hline MYCN copy number & $61(92.4 \%)$ & $88(97.8 \%)$ & 0.111 \\
\hline Loss of Heterozygosity & $23(56.1 \%)$ & $40(90.9 \%)$ & $<0.05$ \\
\hline Tumor Ploidy & $29(58.0 \%)$ & $54(88.5 \%)$ & $<0.05$ \\
\cline { 2 - 4 } & & &
\end{tabular}


TABLE 3: Secondary outcomes

\section{Percutaneous Core Needle Biopsy Incisional Biopsy}

p-value

$$
n=70 \quad n=94
$$

\begin{tabular}{|c|c|c|c|}
\hline Complications, $n(\%)$ & $2(2.90 \%)$ & $3(3.33 \%)$ & 1.00 \\
\hline Transfusion Required, $n$ (\%) & $11(15.7 \%)$ & $38(40.4 \%)$ & 0.001 \\
\hline Any Analgesia Required, $n$ (\%) & $57(86.4 \%)$ & $77(97.5 \%)$ & 0.023 \\
\hline Opiates Required, $n$ (\%) & $43(63.6 \%)$ & $66(83.5 \%)$ & 0.006 \\
\hline$P C A^{a}$ required, $n(\%)$ & $7(10.6 \%)$ & $22(27.9 \%)$ & 0.010 \\
\hline Repeat Biopsy Required, $n$ (\%) & $5(7.1 \%)$ & $1(1.1 \%)$ & 0.085 \\
\hline Admission Post-procedure, $n$ (\%) & $56(80.0 \%)$ & $91(96.8 \%)$ & 0.001 \\
\hline$N P O^{b}$ Duration (median days $\left(I Q R^{c}\right)$ ) & $0(0-0)$ & $0(0-1)$ & 0.559 \\
\hline Length of Stay (median days $\left.\left(I Q R^{c}\right)\right)$ & $8(3.5-22)$ & $10(6-19)$ & 0.607 \\
\hline
\end{tabular}

${ }^{a}$ Patient Controlled Analgesia; ${ }^{b}$ Nil per Os; ${ }^{c}$ Interquartile Range 
TABLE 4: Personnel

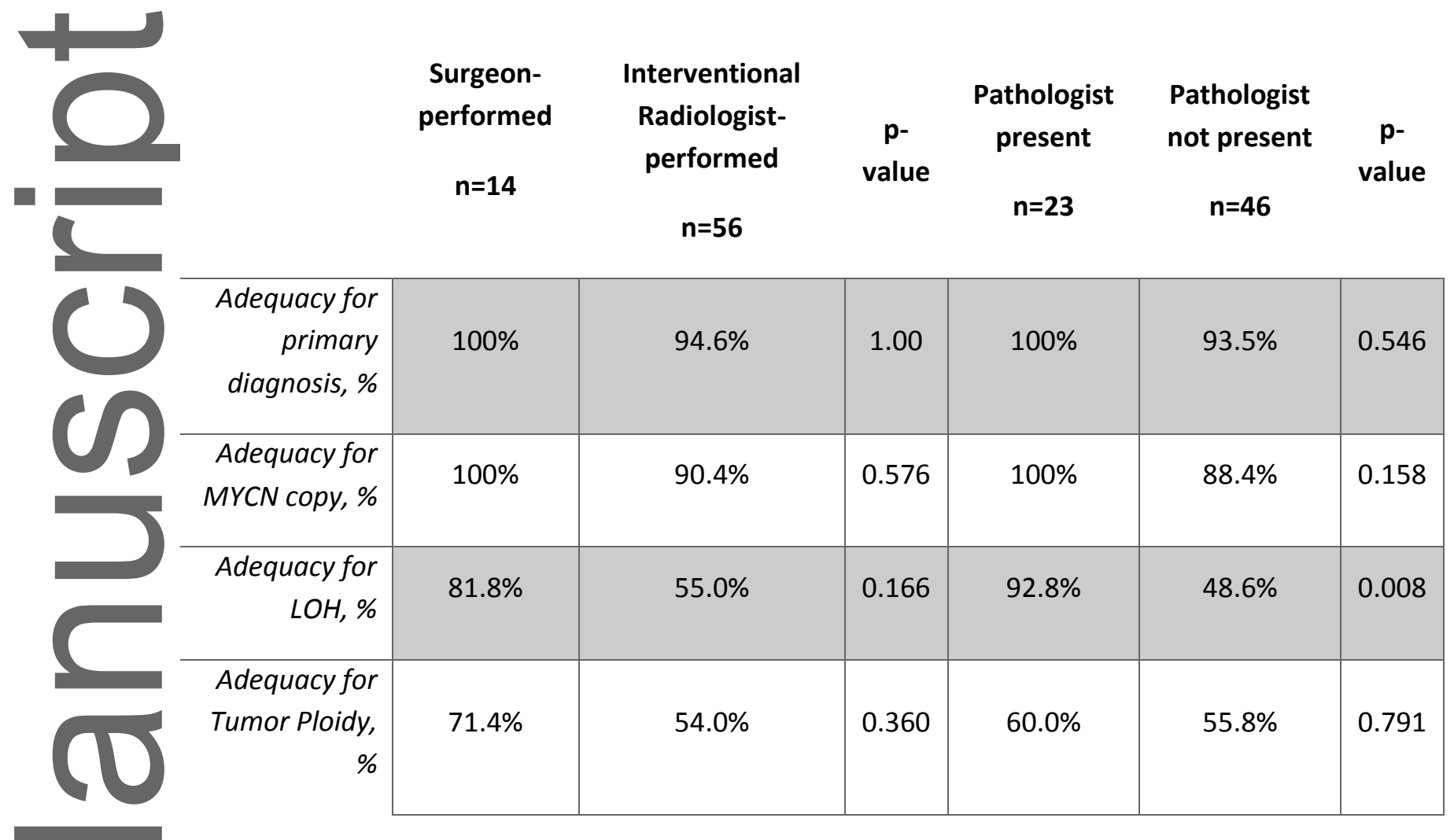

